\title{
Investigation of Helicon discharges as RF coupling concept of negative hydrogen ion sources
}

\author{
S. Briefi ${ }^{* \dagger}$ and U. Fantz ${ }^{* \dagger}$ \\ ${ }^{*}$ Max-Planck-Institut für Plasmaphysik, EURATOM-Assoziation, Boltzmannstr. 2, 85748 \\ Garching, Germany \\ ${ }^{\dagger}$ AG Experimentelle Plasmaphysik, Institut für Physik, Universität Augsburg, 86135 Augsburg, \\ Germany
}

\begin{abstract}
The ITER reference source for $\mathrm{H}^{-}$and $\mathrm{D}^{-}$requires a high RF input power (up to $90 \mathrm{~kW}$ per driver). To reduce the demands on the RF circuit, it is highly desirable to reduce the power consumption while retaining the values of the relevant plasma parameters namely the positive ion density and the atomic hydrogen density. Helicon plasmas are a promising alternative RF coupling concept but they are typically generated in long thin discharge tubes using rare gases and an RF frequency of $13.56 \mathrm{MHz}$. Hence the applicability to the ITER reference source geometry, frequency and the utilization of hydrogen/deuterium has to be proved. In this paper the strategy of the approach for using Helicon discharges for ITER reference source parameters is introduced and the first promising measurements which were carried out at a small laboratory experiment are presented. With increasing RF power a mode transition to the Helicon regime was observed for argon and argon/hydrogen mixtures. In pure hydrogen/deuterium the mode transition could not yet be achieved as the available RF power is too low. In deuterium a special feature of Helicon discharges, the socalled low field peak, could be observed at a moderate B-field of $3 \mathrm{mT}$.
\end{abstract}

Keywords: neutral beam injection, negative hydrogen ion source, helicon discharge

PACS: $29.25 . \mathrm{Ni}, 52.50 . \mathrm{Qt}, 52.70 . \mathrm{Kz}$

\section{INTRODUCTION}

Sources of negative hydrogen ions for fusion applications are currently based on inductive RF coupling. The ITER reference source for $\mathrm{H}^{-}$and $\mathrm{D}^{-}$operates at a pressure of $0.3 \mathrm{~Pa}$ and an RF frequency of $1 \mathrm{MHz}$. High powers of up to $90 \mathrm{~kW}$ are required to generate the plasma in the driver [1]. It is highly desirable to reduce the required RF power while retaining the values of the electron and atomic hydrogen/deuterium densities in order to have more operational margin and reliability and/or cost reduction. The plasma parameters $n_{e}$ and $n_{H} / n_{D}$ are important as they relate closely to the ion and atomic hydrogen/deuterium fluxes towards the plasma grid surface which in turn determine the produced amount of negative hydrogen/deuterium ions at the converter surface.

A very efficient RF coupling mechanism is the Helicon concept which utilizes a special RF antenna geometry together with an external magnetic field [2]. Helicon coupling also has the advantage that the driver concept of the ion source does not have to be changed. Typically, Helicon plasmas are operated in long thin discharge tubes at an RF frequency of $13.56 \mathrm{MHz}$ utilizing rare gases. The experiments proved that with Helicon discharges electron densities can be achieved that exceed the ones obtained in 
ICP discharges by one order of magnitude at the same RF power [2].

Another positive effect of the application of Helicon coupling could be the operation of the ion source at lower pressure. As Helicon discharges can typically be operated at very low pressures (e. g. $0.03 \mathrm{~Pa}$ [3]) but this must be verified for the ITER reference source setup. If the pressure can be reduced, the over-all efficiency of the NNBI system could be increased as the stripping losses of $\mathrm{H}^{-}$due to collisions with the gas would be reduced. Furthermore, more smoother radial profiles of the plasma parameters are expected with Helicon coupling since the coupling mechanism is based on wave heating of the plasma volume [4] not on collisional heating within the skin depth.

To investigate the applicability of Helicon coupling for generating the driver discharge the strategy is to start at the typical Helicon setup and change the geometry, the RF frequency and the working gas stepwise towards the ITER reference source requirements. The aim is to realize a Helicon discharge in the driver geometry using hydrogen/deuterium that reaches similar values of $n_{e}\left(\approx 5 \times 10^{18} \mathrm{~m}^{-3}\right)$ and $n_{H} / n_{D}(\approx$ $1-3 \times 10^{19} \mathrm{~m}^{-3}$ ) as obtained with the inductive coupling but reducing the necessary RF power considerably.

The paper reports on the first step of the strategy. The measurements have been carried out at a small laboratory experiment in the typical Helicon configuration (long thin discharge tube, RF frequency $13.56 \mathrm{MHz}$ ). The investigations have been focused on the transition from utilizing pure argon towards the operation with hydrogen and the comparison of pure $\mathrm{H}_{2}$ and $\mathrm{D}_{2}$ discharges.

\section{EXPERIMENTAL SETUP AND DIAGNOSTIC METHODS}

A sketch of the experimental setup is shown in figure 1. The discharge vessels with a length of $40 \mathrm{~cm}$ and a diameter of $5 \mathrm{~cm}$ or $10 \mathrm{~cm}$ are integrated in a vacuum setup where the working gases are supplied via mass flow controllers (for comparison dimensions of the reference source driver: diameter of $28 \mathrm{~cm}$ and length of $17 \mathrm{~cm}$ ). The pressure is measured with a capacitive pressure gauge. As RF antenna a Nagoya-type Helicon antenna which couples to the $|m|=1$ Helicon modes is applied [4]. The antenna is connected to the RF generator $(13.56 \mathrm{MHz}$, max. $600 \mathrm{~W})$ via a matching network. The external magnetic field which is directed parallel to the cylindrical axis of the discharge

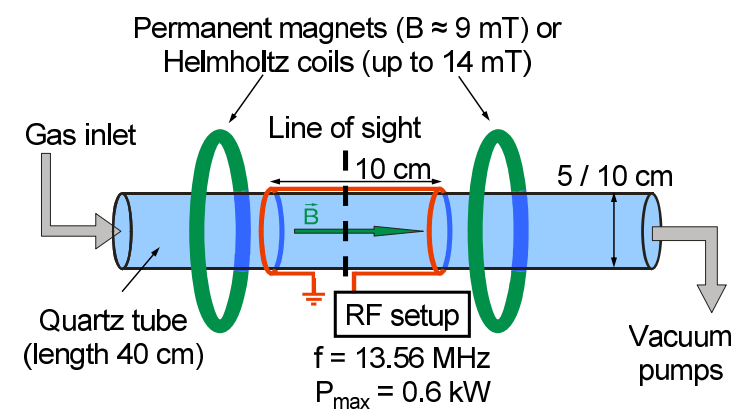

FIGURE 1. Sketch of the experimental setup. 
vessel can be generated by two different methods: either by an array of permanent magnets or by a pair of Helmholtz coils. With the CoSm permanent magnet array (dimensions of the single magnets $9 \times 13 \times 50 \mathrm{~mm}^{3}$ ) a B-field of about $9 \mathrm{mT}$ can be achieved in the antenna region. By utilizing the Helmholtz coils (380 windings and $18 \mathrm{~cm}$ radius each) which produce a much more homogeneous field, the magnetic field strength can be varied between 0 and $14 \mathrm{mT}$ via varying the electrical current through the coils.

Up to now, only optical emission spectroscopy has been utilized as diagnostic method. The measurements were performed with a high-resolution spectrometer $\left(\Delta \lambda_{F W H M} \approx\right.$ $50 \mathrm{pm}$ ) equipped with an ICCD camera. The line of sight was directed radially through the discharge and located in the middle of the RF antenna position. The electron density has been obtained via the line ratio evaluation of argon ion lines $(480.6 \& 488.0 \mathrm{~nm})$ [5] and the electron temperature has been evaluated from the absolute emission of the $750.4 \mathrm{~nm}$ argon line [6]. However, in hydrogen or deuterium only density ratios can be obtained since a similar diagnostic like in argon which does not require additional plasma parameters is not available. The density ratio of atomic to molecular hydrogen/deuterium is determined via an evaluation of the $\mathrm{H}_{2} / \mathrm{D}_{2}$ Blamer lines and the Fulcher system supported by the collisional-radiative models [5].

\section{RESULTS}

\section{Argon and Argon/ $/ \mathrm{H}_{2}\left(\mathrm{D}_{2}\right)$ Discharges}

The investigations presented in this section have all been performed by using the $5 \mathrm{~cm}$ diameter discharge tube and permanent magnets for generating the required external Bfield. Figure 2 shows the electron density determined from the argon ion lines at varying RF power for argon discharges at 0.5 and $1.3 \mathrm{~Pa}$. By increasing the input power a mode transition occurs where the electron density jumps by one order of magnitude. In this high- $n_{e}$ mode intense blue emission form argon ion lines can be observed. This is an indication for Helicon coupling as the "blue mode" has often been reported in Helicon experiments in argon [7] and in the following, this mode is called the Helicon mode. However, the proof that the observed high $-n_{e}$ mode really is the Helicon mode can only be carried out by investigating the time dependent magnetic field in the plasma. This is planned in the near future by applying a $\dot{B}$ probe.

The power required for the mode transition increases with decreasing pressure from $350 \mathrm{~W}$ at $1.3 \mathrm{~Pa}$ to $400 \mathrm{~W}$ at $0.3 \mathrm{~Pa}$. The threshold power also increases with higher hydrogen/deuterium admixture to the argon discharge which can be seen in figure 3 . At a hydrogen/deuterium content of $20 \%$ the required power is about $430 \mathrm{~W}$ (pressure $1.3 \mathrm{~Pa}$ ). For pure hydrogen/deuterium discharges, no mode transition could be observed up to now as the generator power is limited to $600 \mathrm{~W}$.

Figure 4 shows the density ratio of atomic to molecular hydrogen/deuterium and the electron density for different RF powers with a variation of the hydrogen/deuterium content of the argon discharge. If the plasma is in the Helicon regime which is the case for 450 and $550 \mathrm{~W}$ power and a hydrogen/deuterium admixture up to $20 \%$, very high values of $n_{e}$ (up to $4 \times 10^{18} \mathrm{~m}^{-3}$ ) and of the $\mathrm{H} / \mathrm{H}_{2}$ density ratio (up to 1 for 


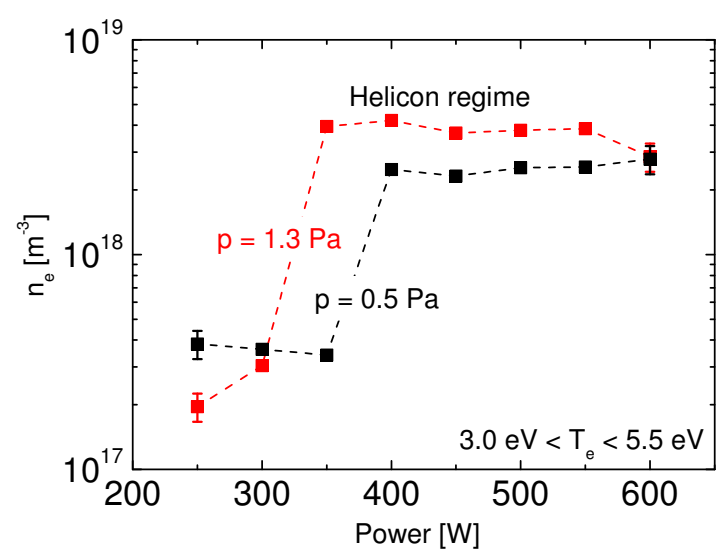

FIGURE 2. Electron density of a pure argon discharge with varying RF power $(5 \mathrm{~cm}$-diameter discharge vessel, permanent magnets).

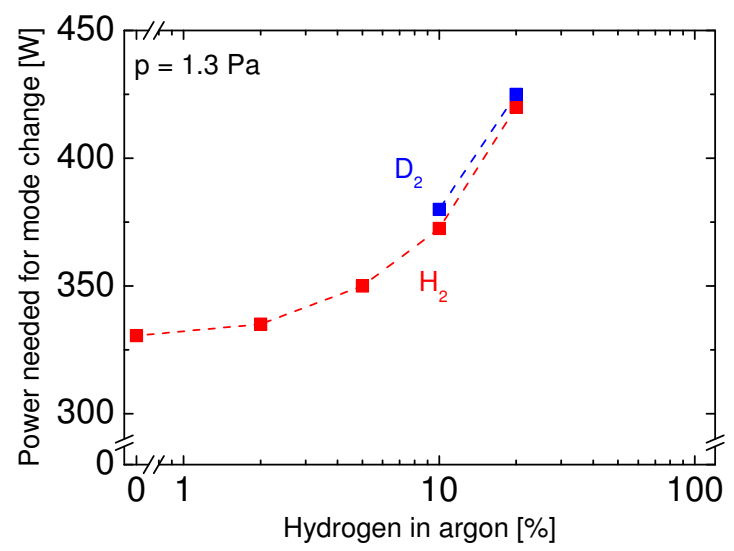

FIGURE 3. RF power required for the mode transition with varying $\mathrm{H}_{2} / \mathrm{D}_{2}$ admixture to argon $(5 \mathrm{~cm}-$ diameter discharge vessel, permanent magnets).

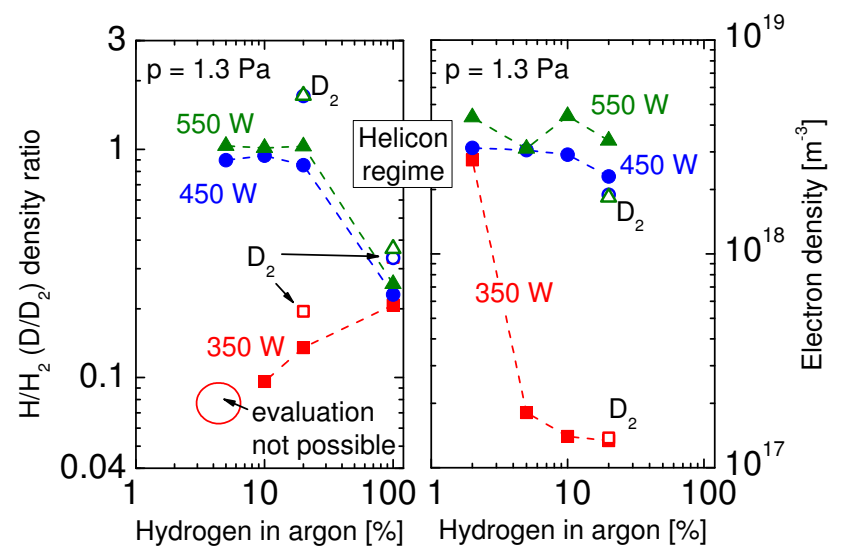

FIGURE 4. Density ratio of atomic to molecular hydrogen/deuterium and electron density with varying $\mathrm{H}_{2} / \mathrm{D}_{2}$ admixture to argon for different $\mathrm{RF}$ powers ( $5 \mathrm{~cm}$-diameter discharge vessel, permanent magnets). 
hydrogen which corresponds to $n_{H}=6.7 \times 10^{19} \mathrm{~m}^{-3}$ and up to 1.7 for deuterium which corresponds to $n_{D}=8.5 \times 10^{19} \mathrm{~m}^{-3}$ ) are obtained. For an RF power of $350 \mathrm{~W}$ the Helicon regime is only reached at very low hydrogen contents.

\section{Pure Hydrogen/Deuterium Discharges}

The measurements presented in this section are carried out with the $10 \mathrm{~cm}$-diameter discharge tube and the Helmholtz coils to allow for a variation of the B-field. As the investigated discharges contain no argon admixture the focus is put on the density ratio of atomic to molecular hydrogen/deuterium.

Figure 5 shows the determined density ratio of atomic to molecular hydrogen (deuterium). In general, the density ratio is higher in deuterium which is commonly observed (also see [8]). If the magnetic field is increased the density ratio increases both for hydrogen and deuterium. However, for deuterium the increase is more pronounced and a so-called low field peak occurs around $3 \mathrm{mT}$ where the density ratio is increased by a factor of 2 compared to the value obtained with no external magnetic field. This peak is typical for Helicon discharges [9]. The peak is observed in all accessible parameters like the discharge emission, the gas temperature and the vibrational temperature of the ground state of the deuterium molecule.

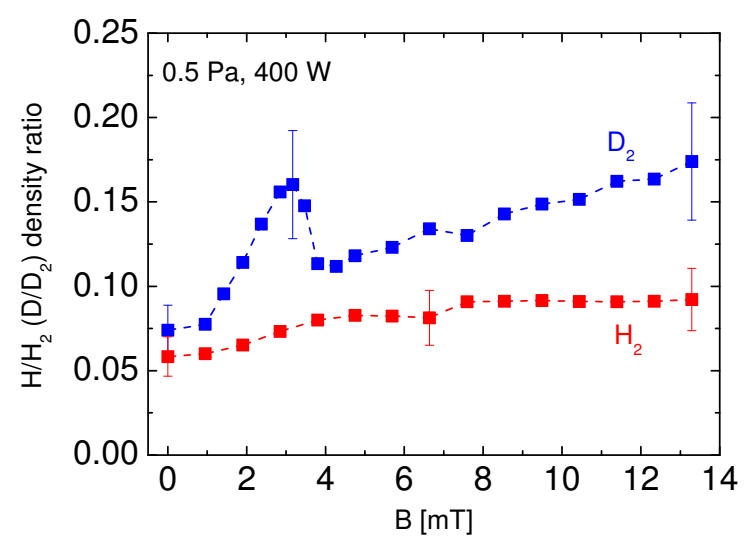

FIGURE 5. Density ratio of atomic to molecular hydrogen/deuterium with varying B-field $(10 \mathrm{~cm}-$ diameter discharge vessel, Helmholtz coils).

If the RF power is increased, also the plasma parameters at the maximum value of the low field peak increase. This is shown exemplarily for the $\mathrm{D} / \mathrm{D}_{2}$ density ratio in figure 6. It should be noted that the B-field required to reach the low field peak maximum is always located at $3 \mathrm{mT}$ independent of the RF power. For comparison, a typical value of the $\mathrm{D} / \mathrm{D}_{2}$ density ratio obtained in the IPP prototype ions source (RF frequency $1 \mathrm{MHz}$, driver diameter $28 \mathrm{~cm}$ ) is also shown in figure 6 . The value is a factor of two larger than the values obtained at the low field peak at an RF power of $600 \mathrm{~W}$ but the RF power is more than a factor 10 higher in the prototype ion source. 


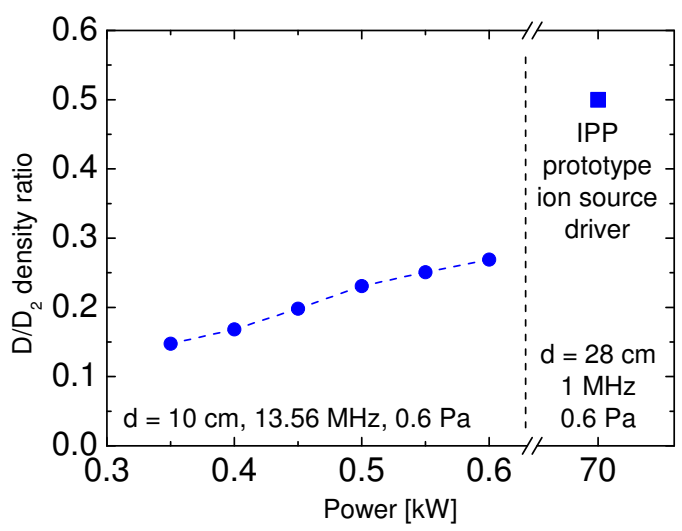

FIGURE 6. Density ratio of atomic to molecular deuterium obtained at the maximum of the low field peak with varying RF power $(10 \mathrm{~cm}$-diameter discharge vessel, Helmholtz coils, $B=3 m T)$. For comparison a typical value of the inductively coupled IPP prototype ion source is also shown.

\section{SUMMARY AND OUTLOOK}

It has been shown, that the Helicon concept provides an efficient RF coupling mechanism not only in the typical setup where rare gases are used but also with hydrogen and deuterium admixtures. The investigations proved that very high electron densities and atomic hydrogen/deuterium densities can be reached with rather low RF powers.

The next step towards the target parameters of the ITER reference source is the utilization of a discharge vessel with a diameter of $20 \mathrm{~cm}$ (length smaller than $40 \mathrm{~cm}$ ) and the transition to an RF frequency of $2 \mathrm{MHz}$ (maximum power $2 \mathrm{~kW}$ ). With the higher available RF power, access to the Helicon regime should also be possible for pure hydrogen/deuterium discharges. In addition the application of a $\dot{B}$ probe for the proof of the existence of Helicon coupling and a double probe for the measurement of ion densities and electron temperatures in pure hydrogen/deuterium discharges is planned.

\section{ACKNOWLEDGMENTS}

The work was supported by an R\&D contract from ITER IO (ITER CT 094100000772 0609). The authors are solely responsible for the content.

\section{REFERENCES}

1. E. Speth, et al., Nuclear Fusion 46, S220 (2006).

2. M. A. Lieberman, and A. J. Lichtenberg, Principles of Plasma Discharges and Materials Processing, John Wiley and Sons New York, 1994.

3. A. Komori, T. Shoji, K. Miyamoto, J. Kawai, and Y. Kawai, Physics of Fluids B: Plasma Physics 3, 893-898 (1991).

4. F. F. Chen, Physics of Plasmas 3, 1783-1793 (1996).

5. U. Fantz, et al., Nuclear Fusion 46, S297 (2006).

6. K. Behringer, Plasma Physics and Controlled Fusion 33, 997 (1991).

7. D. D. Blackwell, and F. F. Chen, Plasma Sources Science and Technology 6, 569 (1997).

8. R. Friedl, Contribution to these conference proceedings (2012).

9. F. F. Chen, Physics of Plasmas 10, 2586-2592 (2003). 